\title{
Parâmetros da marcha em portadores de diabetes mellitus
}

\author{
Gait parameters in patients with diabetes mellitus
}

Cristina Elena Prado Teles Fregonesi ${ }^{1}$ Marcela Regina de Camargo

1 Universidade Estadual Paulista. Programa de Pós-Graduação em Fisioterapia da Faculdade de Ciências e Tecnologia. Presidente Prudente, SP, Brasil.

Recebido em 22/08/08 Revisado em 16/02/09 Aprovado em 24/06/09
Resumo - O Diabetes mellitus é uma enfermidade crônica que leva a alterações sensitivas e motoras. Tais alterações comprometem o equilíbrio e a deambulação, predispondo seus portadores à ocorrência de quedas. Esta revisão teve por objetivo levantar, na literatura recente, estudos que visassem avaliar parâmetros da marcha e aspectos envolvidos com a deambulação. Para isso, foi realizada uma busca nas bases de dados MEDLINE, SciELO, LILACS e PEDro, cruzando as palavras-chave: Neuropatias Diabéticas x Marcha; Diabetes Mellitus x Marcha e Pé Diabético x Marcha. Após passarem pelos critérios de seleção, foram obtidos 15 artigos, os quais foram sintetizados e discutidos, sendo, portanto, incluídos nesta revisão. Ficou claro que a neuropatia diabética leva a déficits na amplitude do passo, velocidade e cadência da marcha em superfícies planas, sem mudanças bruscas de direção ou paradas, e déficits de equilíbrio e coordenação em aclives, declives e terrenos irregulares. Acarreta, também, aumento dos índices de pressão plantar e, devido à alteração de ativação do tríceps sural, dificuldade na fase de apoio terminal e pré-balanço. Assim, o próximo contato inicial ocorrerá de maneira inadequada, com o antepé e sem absorção de choques.

Palavras-chave: Diabetes mellitus; Neuropatias diabéticas; Marcha; Acidentes por quedas.

Abstract - Diabetes mellitus is a chronic disease that results in sensorimotor alterations. These changes affect balance and walking and predispose affected patients to falls. The aim of this review was to identify studies in the recent literature that assess gait parameters and aspects involved in walking. The MEDLINE, SciELO, LILACS and PEDro databases were searched using the following combination of keywords: diabetic neuropathies $x$ gait; diabetes mellitus $x$ gait, and diabetic foot $x$ gait. After the application of selection criteria, 15 articles were retrieved, summarized, discussed, and are included in this review. Diabetic neuropathy was found to lead to deficits in step amplitude, gait velocity and gait cadence on flat surfaces, without sudden changes in direction or stops, and to balance and coordination deficits on inclined and uneven terrain. Diabetic neuropathies also increase plantar pressure rates and lead to difficulties in the terminal stance phase and pre-swing phase due to changes in triceps surae activation. Thus, the next initial contact occurs in an inadequate manner, with the forefoot and without absorption of shocks. Key words: Diabetes mellitus; Diabetic neuropathies; Gait; Accidental falls. 


\section{INTRODUÇÃO}

O Diabetes Mellitus (DM) é uma das enfermidades crônicas mais diagnosticada nos últimos anos. Acomete cerca de 170 milhões de indivíduos no mundo e há uma previsão de aumento de 114\% até 2030. Nos países em desenvolvimento, este aumento será ainda maior, cerca de $146 \%$ em relação aos 115 milhões de diabéticos existentes hoje ${ }^{1}$.

A principal complicação do DM é a neuropatia diabética periférica (NDP), cuja prevalência pode chegar a $50 \%$ dos casos ${ }^{2}$. Este tipo de neuropatia é caracterizado por uma síndrome clínica ou sub-clínica que afeta diferentes tipos de nervos, tendo uma evolução que varia desde manifestações silenciosas até a ocorrência de sinais e sintomas, como perda de sensibilidade somatossensitiva. Seu diagnóstico deve ser feito por exclusão ${ }^{3}$, uma vez que é dado pela presença de sinais e sintomas de disfunção nervosa periférica em portadores de DM, eliminando outras possíveis causas. No entanto, para sua confirmação, um exame clínico detalhado deve ser realizado, pois a ausência de sintomas não pode ser entendida como ausência de neuropatia ${ }^{4}$.

Além do envolvimento das fibras aferentes, com a progressão do quadro, a NDP leva ao comprometimento das fibras nervosas motoras, desencadeando atrofia subjacente à fraqueza muscular. A lesão se dá no sentido distal para proximal, sendo as articulações dos pés e tornozelos primeiramente atingidas 5 . A fisiopatologia da NDP é responsável, ainda, pelo aparecimento de áreas anômalas de pressão nas plantas dos pés, agravadas pelo atraso de ativação de alguns músculos durante a marcha e pelo déficit sensitivo, posto que com os pés anestesiados, os diabéticos não sentem dor. O resultado pode ser o aparecimento de úlceras plantares que, se não tratadas devidamente, levam às amputações ${ }^{6}$.

Em idosos com diabetes, neuropatias periféricas são especialmente problemáticas devido à perda gradual da estabilidade, com decréscimos nas oscilações do centro de pressão, e da função sensóriomotora, com possíveis adaptações compensatórias de mecanismos musculoesqueléticos. A presença de NDP associada à idade avançada culminam em uma condução nervosa deficitária, o que causa um impacto negativo sobre as atividades da vida diária, inclusive na marcha.

O processo natural da senilidade, por si só, já envolve alterações do sistema musculoesquelético e do sistema somatossensitivo, levando ao comprometimento do controle postural ${ }^{10-12}$. Somando-se às alterações nervosas, somatossensitivas e motoras da
$\mathrm{NDP}^{13}$, a marcha deste indivíduo sofre alterações, ocorrendo episódios de desequilíbrios. Por conseguinte, com a progressão do quadro de neuropatia, multiplicam-se os riscos de quedas, que constituem um problema de saúde pública no mundo ${ }^{13-16}$.

Gutierrez et al..$^{14}$, após estudo com 18 mulheres portadoras de diabetes, concluíram que a perda gradual do equilíbrio nesta população pode estar relacionada ao déficit de força muscular de tornozelo encontrado no estudo. Kanade et al. ${ }^{13}$, em um estudo envolvendo 84 diabéticos com alterações relativas à neuropatia, concluíram que a capacidade e o desempenho da marcha diminuem com a progressão das complicações (nervosas e circulatórias) ocorridas nos pés.

Wallace et al. ${ }^{15}$, em estudo com 400 diabéticos, observaram que a prevalência de quedas chega a 1,25 por ano e que ocorreu, pelo menos, um episódio anual de queda em $54 \%$ da população estudada. Maurer et al. ${ }^{16}$, após estudarem 139 idosos, sendo $13 \%$ diabéticos e $87 \%$ não diabéticos, encontraram incidência de quedas em 78\% nos diabéticos e 30\% nos não diabéticos e concluíram que o DM deve ser considerado um fator de risco para quedas e a identificação dos indivíduos nesta situação pode facilitar a tomada de medidas preventivas, destinadas a reduzir o risco de quedas.

Diante do exposto, a presente revisão busca levantar, na literatura, estudos que relatem as características do padrão de marcha e risco de quedas em NDP, bem como, discutir sobre resultados similares e contraditórios destes estudos, para que se possa fornecer orientação sobre o rumo que deve ser dado à realização de futuras pesquisas na área.

\section{DESENVOLVIMENTO}

Em abril de 2008, foi realizada busca abrangendo as bases de dados da área da saúde: MEDLINE; SCieLO; LILACS; e PEDro. Na estratégia, foram cruzados os termos indexados Neuropatias Diabéticas x Marcha; Diabetes Mellitus x Marcha e Pé Diabético x Marcha, nos idiomas ingles e português. As buscas realizadas com todos os cruzamentos nos permitiu encontrar 250 publicações sobre o assunto. Os resultados da busca foram filtrados, sendo somente utilizadas as publicações de janeiro de 2003 a abril de 2008.

No processo de seleção, foi realizada leitura de todos os resumos e optado por incluir nesta revisão apenas artigos relacionados a ensaios clínicos para avaliação das características da marcha de diabéticos. Foram excluídos artigos de revisão, relatos 
de casos e comunicações breves. Em relação ao perfil da amostra, foram selecionados artigos que incluíssem portadores de diabetes tipo I ou II, com ausência de amputações, deformidades ortopédicas e que deambulassem sem assistência. Após este procedimento, foram incluídos, nesta revisão, 15 artigos originais, todos na língua inglesa e escritos por 13 diferentes primeiro autores (Figura 1).

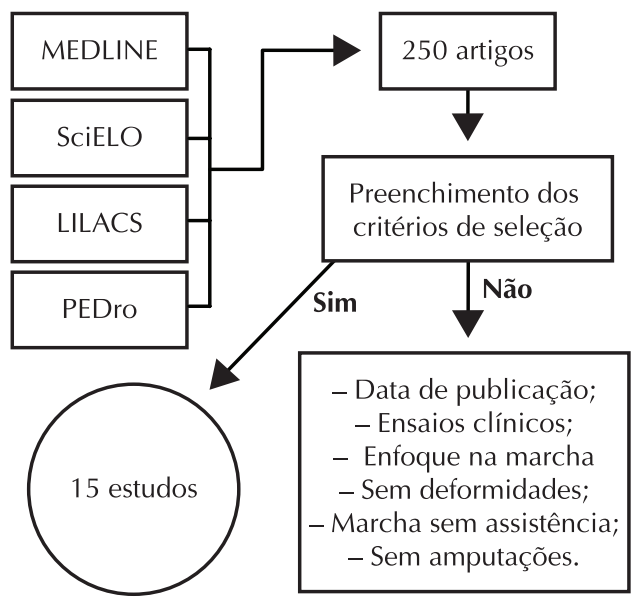

Figura 1. Metodologia para seleção dos artigos incluídos no estudo.

O quadro 1 agrupa os artigos incluídos e sintetiza as principais informações de cada um e utiliza as seguintes abreviaturas:

\section{- Amostra}

CO: Controles; D: Diabéticos; NDP: Neuropatas diabéticos; NDU: Neuropatas diabéticos ulcerados; NDQ: Neuropatas diabéticos que já sofreram quedas; N/C: Não consta.

- Critérios de elaboração do perfil da amostra DM: Diabetes Mellitus; MNSI: Michigan Neuropathy Screening Instrument; NDS: Neuropathy Disability Score; VPT: Limiar de sensibilidade vibratória; TPS: Teste de sensibilidade somatossensitiva; ND: Neuropatia diabética; MMII: Membros inferiores; ADM: Amplitude de movimento; FM: força muscular; EMG: Eletromiografia.

\section{- Parâmetros avaliados}

US: Ultrassom; GRF: Força de reação ao solo.

\section{- Conclusões}

MTF: Articulação metatarsofalângica; AP:

Ântero-posterior; ML: Médio-lateral.

Na coluna "Amostra" são descritos o número e o tipo de população incluída. Onze estudos ${ }^{17-23,26,27,29,31}$ incluem portadores de NDP, sete ${ }^{18,24,25,28-31}$ incluem diabéticos sem neuropatia, três estudos ${ }^{17,18,29}$ incluem NDP que já tiveram úlceras, um estudo ${ }^{27}$ inclui um grupo NDP que já sofreu queda e onze estudos ${ }^{17-1,92-2-25,28-31}$ foram controlados com indivíduos não-diabéticos.

Quatro estudos ${ }^{24,25,28,30}$ não incluíram NDP, comparando um grupo diabético sem neuropatia com controle não-diabéticos.

Na coluna "Critérios de elaboração do perfil da amostra", são descritos os parâmetros de inclusão e exclusão dos estudos para eliminação de possíveis variáveis que causem confusão. Dez estudos ${ }^{17-19,21-23,26,27,29,30}$ descreveram algum critério para confirmação de NDP, sete ${ }^{17,18,22,23,26-28}$ descreveram a exclusão de amputados, seis $s^{17,18,25-28}$ tiveram critérios relacionados à idade, seis $^{17-19,22,24,25}$ excluíram portadores de enfermidades ortopédicas, dez 17-19,22,23,26-29,31 excluíram portadores de doenças neurológicas, seis ${ }^{18,19,26,27,29,31}$ excluíram portadores de moléstias musculoesqueléticas, cinco $^{17,23,26-28}$ descreveram a exclusão de dependentes de assistência para deambulação e quatro ${ }^{17,28,30,31}$ excluíram indivíduos portadores de úlceras no momento da avaliação.

Nas colunas "Proporção entre os sexos", "Média ou mínino e máximo de idade" " "IMC" estão relacio-

Quadro 1. Artigos incluídos no estudo e suas principais informações.

\begin{tabular}{|c|c|c|c|c|c|c|c|}
\hline Estudo & \begin{tabular}{|l} 
Amos- \\
tra [n] \\
(DM \\
Tipo 1 / \\
Tipo 2)
\end{tabular} & $\begin{array}{l}\text { Critérios de elabo- } \\
\text { ração do perfil da } \\
\text { amostra }\end{array}$ & $\begin{array}{c}\text { Propor- } \\
\text { ção entre } \\
\text { os sexos } \\
\text { (masculino/ } \\
\text { feminino) }\end{array}$ & $\begin{array}{l}\text { Média(dp) } \\
\text { ou mínimo - } \\
\text { máximo de } \\
\text { idade. }\end{array}$ & $\begin{array}{l}\text { IMC (Índice de } \\
\text { massa corpó- } \\
\text { rea) médio }\end{array}$ & $\begin{array}{l}\text { Parâmetros } \\
\text { avaliados }\end{array}$ & Conclusões \\
\hline $\begin{array}{l}\text { Akashi et } \\
\text { al. }{ }^{7}\end{array}$ & \begin{tabular}{|l}
{$[45]$} \\
$19 N D P$ \\
$(0 / 19)$ \\
$10 N D U$ \\
$(0 / 10)$ \\
$16 C O$
\end{tabular} & $\begin{array}{l}\text { Incluídos: } 5 \text { anos de } \\
\text { DM; e pontuação } \\
\text { de } 6 \text { ou mais no } \\
\text { MNSI. Excluídos: } \\
\text { amputações, idade } \\
\text { superior a } 65 \text { anos, } \\
\text { doença ortopédica } \\
\text { ou neurológica do } \\
\text { pé, dependência } \\
\text { para deambulação, } \\
\text { presença de úlceras } \\
\text { durante a avaliação } \\
\text { e abuso de álcool. }\end{array}$ & $\begin{array}{l}\text { 11/8NDP } \\
\text { 5/5NDU } \\
\text { 8/8CO }\end{array}$ & $\begin{array}{l}57,6(8,5) \text { NDP } \\
53,8(7,9) \text { NDU } \\
51,1(8,3) \mathrm{CO}\end{array}$ & $\begin{array}{l}\text { 26,6(4,2)NDP } \\
27,8(4,6) \mathrm{NDU} \\
23,9(2,9) \mathrm{CO}\end{array}$ & $\begin{array}{l}\text { EMG e GRF: } \\
\text { Tibial anterior, } \\
\text { vasto lateral e } \\
\text { gastrocnêmio } \\
\text { lateral durante } \\
\text { fase de apoio } \\
\text { numa platafor- } \\
\text { ma de força. }\end{array}$ & $\begin{array}{l}\text { Atraso na ativa- } \\
\text { ção do gastroc- } \\
\text { nêmio e vastos } \\
\text { laterais, em NDU, } \\
\text { diminuindo a } \\
\text { proporção do } \\
\text { momento vertical } \\
\text { durante a fase de } \\
\text { propulsão que } \\
\text { pode comprome- } \\
\text { ter a capacidade } \\
\text { de marcha. }\end{array}$ \\
\hline
\end{tabular}




\begin{tabular}{|c|c|c|c|c|c|c|c|}
\hline Estudo & \begin{tabular}{|} 
Amos- \\
tra [n] \\
(DM \\
Tipo 1/ \\
Tipo 2)
\end{tabular} & $\begin{array}{c}\text { Critérios de elabo- } \\
\text { ração do perfil da } \\
\text { amostra }\end{array}$ & $\begin{array}{l}\text { Propor- } \\
\text { ção entre } \\
\text { os sexos } \\
\text { (masculino/ } \\
\text { feminino) }\end{array}$ & $\begin{array}{l}\text { Média(dp) } \\
\text { ou mínimo - } \\
\text { máximo de } \\
\text { idade. }\end{array}$ & $\begin{array}{l}\text { IMC (Índice de } \\
\text { massa corpó- } \\
\text { rea) médio }\end{array}$ & $\begin{array}{l}\text { Parâmetros } \\
\text { avaliados }\end{array}$ & Conclusões \\
\hline $\begin{array}{l}\text { D'Ambrogi } \\
\text { et al. }{ }^{18}\end{array}$ & \begin{tabular}{|l|}
{$[82]$} \\
$19 N D P$ \\
$(9 / 10)$ \\
$15 N D U$ \\
$(3 / 12)$ \\
$27 D$ \\
$(8 / 19)$ \\
$21 C O$
\end{tabular} & $\begin{array}{l}\text { Incluídos em } \\
\text { NDP/NDU: pontu- } \\
\text { ação >5 no NDS e } \\
\text { >25V no VPT. In- } \\
\text { cluídos em NDU: } \\
\text { história prévia de } \\
\text { úlcera nas cabe- } \\
\text { ças dos metatarsos } \\
3 \text { meses antes do } \\
\text { estudo. Excluídos: } \\
\text { idade superior a } \\
70 \text { anos, presença } \\
\text { de outras doenças } \\
\text { que culminassem } \\
\text { no quadro, ampu- } \\
\text { tações. }\end{array}$ & $\begin{array}{l}\text { 10/9NDP } \\
10 / 5 \mathrm{NDU} \\
19 / 8 \mathrm{D} \\
13 / 8 \mathrm{CO}\end{array}$ & $\begin{array}{l}53,7(10,4) \mathrm{NDP} \\
57,3(9,6) \mathrm{NDU} \\
52,7(12,7) \mathrm{D} \\
56,6(11,8) \mathrm{CO}\end{array}$ & $\begin{array}{l}27,0(4,9) \mathrm{NDP} \\
27,5(4,1) \mathrm{NDU} \\
25,3(3,4) \mathrm{D} \\
25,0(3,1) \mathrm{CO}\end{array}$ & $\begin{array}{l}\text { US e GRF: } \\
\text { Espessamento } \\
\text { do tendão de } \\
\text { Aquiles e da } \\
\text { fáscia plantar e } \\
\text { marcha numa } \\
\text { plataforma de } \\
\text { força. }\end{array}$ & $\begin{array}{l}\text { Aumento no } \\
\text { espessamento } \\
\text { do tendão de } \\
\text { Aquiles e da } \\
\text { fáscia plantar, } \\
\text { presente na } \\
\text { neuropatia, leva } \\
\text { a permanência } \\
\text { do mecanismo } \\
\text { de Windlass du- } \\
\text { rante todo ciclo } \\
\text { de marcha. }\end{array}$ \\
\hline Gates et al. ${ }^{19}$ & $\begin{array}{l}{[26]} \\
14 \mathrm{NDP} \\
(\mathrm{N} / \mathrm{C}) \\
12 \mathrm{CO}\end{array}$ & $\begin{array}{l}\text { Incluídos: } \\
\text { IMC>25, ausência } \\
\text { de medicações, } \\
\text { cirurgias, lesões } \\
\text { ou doenças que } \\
\text { impedissem a } \\
\text { marcha e resposta } \\
\text { positiva ao TPS. }\end{array}$ & $\begin{array}{l}\text { 12/2NDP } \\
10 / 2 \mathrm{CO}\end{array}$ & $\begin{array}{l}61,0(6,6) \mathrm{NDP} \\
57,6(7,7) \mathrm{CO}\end{array}$ & $\begin{array}{l}30,3(4,4) \mathrm{NDP} \\
29,4(2,2) \mathrm{CO}\end{array}$ & $\begin{array}{l}\text { Eletro-goniô- } \\
\text { metro: ADM } \\
\text { de joelhos, } \\
\text { cadência e } \\
\text { velocidade du- } \\
\text { rante a marcha } \\
\text { fisiológica. }\end{array}$ & $\begin{array}{l}\text { Geração e } \\
\text { regulação do } \\
\text { intervalo de pas- } \\
\text { sos permanecem } \\
\text { inalteradas em } \\
\text { NDP. }\end{array}$ \\
\hline $\begin{array}{l}\text { Guldemond } \\
\text { et al. }{ }^{20}\end{array}$ & $\begin{array}{l}{[20]} \\
20 \mathrm{NDP} \\
(\mathrm{N} / \mathrm{C})\end{array}$ & $\begin{array}{l}\text { Incluídos: pés } \\
\text { neuropáticos não } \\
\text { deformados e com } \\
\text { altos índices de } \\
\text { pressão plantar. }\end{array}$ & 17/ONDP & 44-78NDP & $19,3-46 N D P$ & \begin{tabular}{|l|} 
Palmilha para \\
detecção de \\
índices de \\
pressão plantar \\
e palmilha de \\
posicionamento \\
podal durante \\
a marcha em \\
esteira.
\end{tabular} & $\begin{array}{l}\text { A combinação } \\
\text { de uma cúpula } \\
\text { e de um apoio } \\
\text { suplementar leva } \\
\text { à diminuição } \\
\text { dos índices de } \\
\text { pressão plantar } \\
\text { durante a mar- } \\
\text { cha. }\end{array}$ \\
\hline Hsi et al. ${ }^{21}$ & $\begin{array}{l}{[10]} \\
10 \mathrm{NDP} \\
(\mathrm{N} / \mathrm{C})\end{array}$ & $\begin{array}{l}\text { Incluídos: incapa- } \\
\text { cidade de sentir } \\
\text { o monofilamento } \\
5.07 \text { e sem história } \\
\text { prévia de ulcera- } \\
\text { ção. }\end{array}$ & $3 / 7 \mathrm{NDP}$ & 63(9)NDP & - & $\begin{array}{l}\text { Palmilha para } \\
\text { detecção de } \\
\text { pressão plantar } \\
\text { e "rocker-sole" } \\
\text { durante marcha } \\
\text { fisiológica. }\end{array}$ & $\begin{array}{l}\text { Calçados com } \\
\text { "rocker-sole" } \\
\text { diminuem os ín- } \\
\text { dices de pressão } \\
\text { plantar levando } \\
\text { sua redistribui- } \\
\text { ção durante a } \\
\text { marcha. }\end{array}$ \\
\hline Kwon et al. ${ }^{22}$ & $\begin{array}{l}{[18]} \\
09 \mathrm{NDP} \\
(2 / 7) \\
09 \mathrm{CO}\end{array}$ & $\begin{array}{l}\text { Incluídos: insensi- } \\
\text { bilidade ao mono- } \\
\text { filamento } 5.07 \text { e } \\
\text { história de úlcera } \\
\text { plantar. Excluídos: } \\
\text { demência, am- } \\
\text { putação, doença } \\
\text { neurológica ou } \\
\text { ortopédica. }\end{array}$ & $\begin{array}{l}\text { 6/3NDP } \\
6 / 3 \mathrm{CO}\end{array}$ & $\begin{array}{l}59,2(11,2) \mathrm{NDP} \\
59,2(12,6) \mathrm{CO}\end{array}$ & - & $\begin{array}{l}\text { Fotogrametria } \\
\text { ADM (dorsi- } \\
\text { flexão com jo- } \\
\text { elhos fletidos, } \\
\text { dorsiflexão } \\
\text { com joelhos } \\
\text { estendidos, } \\
\text { eversão e } \\
\text { inversão) e } \\
\text { EMG do vasto } \\
\text { lateral, sóleo, } \\
\text { gastrocnêmio } \\
\text { medial, e } \\
\text { ísquiotibial } \\
\text { medial e velo- } \\
\text { cidade durante } \\
\text { marcha em } \\
\text { superfície } \\
\text { plana. }\end{array}$ & $\begin{array}{l}\text { Ativação precoce } \\
\text { do sóleo e do } \\
\text { gastrocnêmio } \\
\text { medial leva a um } \\
\text { contato inicial } \\
\text { prematuro do } \\
\text { antepé com o } \\
\text { solo, diminuindo } \\
\text { a absorção de } \\
\text { impacto e acen- } \\
\text { tuando a fase de } \\
\text { apoio da marcha. }\end{array}$ \\
\hline
\end{tabular}




\begin{tabular}{|c|c|c|c|c|c|c|c|}
\hline Estudo & $\begin{array}{c}\text { Amos- } \\
\text { tra [n] } \\
\text { (DM } \\
\text { Tipo 1 / } \\
\text { Tipo 2) }\end{array}$ & $\begin{array}{l}\text { Critérios de elabo- } \\
\text { ração do perfil da } \\
\text { amostra }\end{array}$ & $\begin{array}{c}\text { Propor- } \\
\text { ção entre } \\
\text { Os sexos } \\
\text { (masculino/ } \\
\text { feminino) }\end{array}$ & $\begin{array}{l}\text { Média(dp) } \\
\text { ou mínimo-- } \\
\text { máximo de } \\
\text { idade. }\end{array}$ & $\begin{array}{l}\text { IMC (Índice de } \\
\text { massa corpó- } \\
\text { rea) médio }\end{array}$ & $\begin{array}{l}\text { Parâmetros } \\
\text { avaliados }\end{array}$ & Conclusões \\
\hline Menz et al. ${ }^{23}$ & $\begin{array}{l}{[60]} \\
30 \mathrm{NDP} \\
(0 / 30) \\
30 \mathrm{CO}\end{array}$ & \begin{tabular}{|l|} 
Incluídos: VPT e \\
teste de sensibilida- \\
de tátil. Excluídos: \\
doença de Parkin- \\
son, amputação, \\
grande cirurgia no \\
pé, deformidades \\
ortopédicas e \\
marcha com assis- \\
tência.
\end{tabular} & $\begin{array}{l}\text { 22/8NDP } \\
22 / 8 \mathrm{CO}\end{array}$ & $\begin{array}{l}\text { 55-91NDP } \\
55-91 \mathrm{CO}\end{array}$ & & $\begin{array}{l}\text { Cadência e } \\
\text { velocidade da } \\
\text { marcha, ampli- } \\
\text { tude do passo, } \\
\text { acelerometria } \\
\text { da cabeça e } \\
\text { da pelve du- } \\
\text { rante marcha } \\
\text { em terreno } \\
\text { irregular. }\end{array}$ & $\begin{array}{l}\text { Os NDP demons- } \\
\text { tram diferenças } \\
\text { claras entre a } \\
\text { estabilidade da } \\
\text { cabeça e pelve } \\
\text { durante a acele- } \\
\text { ração, adotando } \\
\text { um padrão de } \\
\text { marcha mais } \\
\text { conservador. }\end{array}$ \\
\hline $\begin{array}{l}\text { Petrofsky et } \\
\text { al. }{ }^{24}\end{array}$ & $\begin{array}{l}{[55]} \\
25 \mathrm{D} \\
(0 / 25) \\
30 \mathrm{CO}\end{array}$ & $\begin{array}{l}\text { Incluídos CO: } \\
\text { ausência de DM. } \\
\text { Excluídos: incapa- } \\
\text { cidade de sentir } \\
\text { 10g de pressão, } \\
\text { diminuição da FM e } \\
\text { doenças ortopédi- } \\
\text { cas ou articulares } \\
\text { nos MMII. }\end{array}$ & $\begin{array}{l}\text { 13/12D } \\
19 / 11 \mathrm{CO}\end{array}$ & $\begin{array}{l}61,6(8,6) \mathrm{D} \\
43,2(19,5) \mathrm{CO}\end{array}$ & $\begin{array}{l}28,4(6,0) \mathrm{D} \\
22,3(3,5) \mathrm{CO}\end{array}$ & $\begin{array}{l}\text { Cadência e } \\
\text { velocidade da } \\
\text { marcha: acele- } \\
\text { rometria EMG: } \\
\text { gastrocnêmio } \\
\text { medial e tibial } \\
\text { anterior. } \\
\text { Sensores plan- } \\
\text { tares durante a } \\
\text { marcha. }\end{array}$ & $\begin{array}{l}\text { Em D a marcha } \\
\text { está comprome- } \\
\text { tida com passos } \\
\text { incertos e ampli- } \\
\text { tude aumentada. }\end{array}$ \\
\hline $\begin{array}{l}\text { Petrofsky et } \\
\text { al. }^{25}\end{array}$ & $\begin{array}{l}{[31]} \\
15 \mathrm{D} \\
(0 / 15) \\
16 \mathrm{CO}\end{array}$ & $\begin{array}{l}\text { Incluídos: } 5 \text { anos } \\
\text { de DM (glicose } \\
\text { sérica }>126 \mathrm{mg} \text { ), } \\
\text { idade entre } 40-70 \\
\text { anos, sem história } \\
\text { de quedas, doenças } \\
\text { ortopédicas ou } \\
\text { articulares. Excluí- } \\
\text { dos: déficit de FM, } \\
\text { sensibilidade ou } \\
\text { ADM. }\end{array}$ & $\begin{array}{l}8 / 8 \mathrm{D} \\
7 / 8 \mathrm{CO}\end{array}$ & $\begin{array}{l}57(5,1) \mathrm{D} \\
56(6,3) \mathrm{CO}\end{array}$ & - & $\begin{array}{l}\text { Cadência e } \\
\text { velocidade da } \\
\text { marcha: acele- } \\
\text { rometria } \\
\text { Índices de } \\
\text { pressão plan- } \\
\text { tar: palmilha } \\
\text { transdutora. }\end{array}$ & $\begin{array}{l}\text { Diabéticos apre- } \\
\text { sentam distúrbios } \\
\text { na marcha e ris- } \\
\text { cos para queda, } \\
\text { anteriormente as } \\
\text { perdas sensiti- } \\
\text { vas. A perda do } \\
\text { equilíbrio leva à } \\
\text { redistribuição de } \\
\text { pressões nos pés. }\end{array}$ \\
\hline $\begin{array}{l}\text { Richardson } \\
\text { et al. }{ }^{26}\end{array}$ & $\begin{array}{l}{[42]} \\
42 \mathrm{NDP} \\
(\mathrm{N} / \mathrm{C})\end{array}$ & \begin{tabular}{|l|} 
Incluídos: idade \\
entre 45-85 anos, \\
sintomas de NDP, \\
deambulação sem \\
assistência, falar \\
e entender inglês, \\
sinais clínicos e \\
no EMG de NDP. \\
Excluídos: peso \\
>136Kg, doenças \\
neurológicas, \\
músculoesqueléti- \\
cas amputações, \\
EMG de outras \\
doenças.
\end{tabular} & 22/20NDP & $65,9(10,4) \mathrm{NDP}$ & $32,1(6,9) \mathrm{NDP}$ & $\begin{array}{l}\text { Largura, com- } \\
\text { primento do } \\
\text { passo, velocida- } \\
\text { de da marcha } \\
\text { e introdução } \\
\text { de métodos } \\
\text { assistivos. }\end{array}$ & $\begin{array}{l}\text { A inserção } \\
\text { de métodos } \\
\text { assistivos pode } \\
\text { colaborar para } \\
\text { evitar o risco } \\
\text { de quedas em } \\
\text { pacientes diabé- } \\
\text { ticos. }\end{array}$ \\
\hline $\begin{array}{l}\text { Richardson } \\
\text { et al. } .^{27}\end{array}$ & $\begin{array}{l}{[42]} \\
22 \mathrm{NDQ} \\
(\mathrm{N} / \mathrm{C}) \\
20 \mathrm{NDP} \\
(\mathrm{N} / \mathrm{C})\end{array}$ & \begin{tabular}{|l|} 
Incluídos: idade \\
entre 50-85 anos, \\
sintomas de NDP, \\
deambulação sem \\
assistência, falar \\
e entender inglês, \\
sinais clínicos e \\
EMG de NDP. \\
Excluídos: peso \\
>136Kg, enfermida- \\
des neurológicas, \\
musculoesqueléticas \\
distúrbio de equi- \\
líbrio, amputação, \\
respostas anormais a \\
testes neurológicos.
\end{tabular} & $\begin{array}{l}\text { 8/14NDQ } \\
6 / 14 \mathrm{NDP}\end{array}$ & $64,7(9,8) \mathrm{NDP}$ & $31,8(6,6) \mathrm{NDP}$ & $\begin{array}{l}\text { Largura, } \\
\text { comprimen- } \\
\text { to do passo, } \\
\text { velocidade da } \\
\text { marcha. }\end{array}$ & $\begin{array}{l}\text { Em superfícies } \\
\text { irregulares, NDQ } \\
\text { tem maior varia- } \\
\text { bilidade do passo } \\
\text { e adaptabilidade } \\
\text { do andar que } \\
\text { NDP. }\end{array}$ \\
\hline
\end{tabular}

Continua 


\begin{tabular}{|c|c|c|c|c|c|c|c|}
\hline Estudo & $\begin{array}{l}\text { Amos- } \\
\text { tra [n] } \\
\text { (DM } \\
\text { Tipo 1/ } \\
\text { Tipo 2) }\end{array}$ & $\begin{array}{l}\text { Critérios de elabo- } \\
\text { ração do perfil da } \\
\text { amostra }\end{array}$ & $\begin{array}{l}\text { Propor- } \\
\text { ção entre } \\
\text { OS sexos } \\
\text { (masculino/ } \\
\text { feminino) }\end{array}$ & $\begin{array}{l}\text { Média(dp) } \\
\text { ou mínimo - } \\
\text { máximo de } \\
\text { idade. }\end{array}$ & $\begin{array}{l}\text { IMC (Índice de } \\
\text { massa corpó- } \\
\text { rea) médio }\end{array}$ & $\begin{array}{c}\text { Parâmetros } \\
\text { avaliados }\end{array}$ & Conclusões \\
\hline Sacco et al. ${ }^{28}$ & $\begin{array}{l}{[40]} \\
20 D \\
(0 / 20) \\
20 C O\end{array}$ & $\begin{array}{l}\text { Excluídos: idade } \\
\text { >65 anos, presença } \\
\text { de úlcera plantar } \\
\text { durante a avaliação, } \\
\text { uso de assistência } \\
\text { para andar, vesti- } \\
\text { bulopatia, doença } \\
\text { vascular periférica, } \\
\text { enfermidade mus- } \\
\text { cular, reumática, } \\
\text { neurológica ou } \\
\text { visual, uso abusivo } \\
\text { de álcool e ampu- } \\
\text { tações. }\end{array}$ & $\begin{array}{l} \\
8 / 8 \mathrm{D} \\
12 / 8 \mathrm{CO}\end{array}$ & $\begin{array}{l}51,5(11,2) \mathrm{D} \\
40,1(10,4) \mathrm{CO}\end{array}$ & - & $\begin{array}{l}\text { EMG: tibial } \\
\text { anterior, vasto } \\
\text { lateral, gastroc- } \\
\text { nêmio lateral e } \\
\text { respectivos } \\
\text { GRF. }\end{array}$ & $\begin{array}{l}\text { Além dos danos } \\
\text { dos recursos } \\
\text { sensitivos e } \\
\text { motores, a NDP } \\
\text { leva a alterações } \\
\text { do mecanismo } \\
\text { intrínseco de } \\
\text { controle motor } \\
\text { ineficiência } \\
\text { da articulação } \\
\text { do tornozelo o } \\
\text { que gera danos } \\
\text { no equilíbrio e } \\
\text { progressão da } \\
\text { marcha. }\end{array}$ \\
\hline $\begin{array}{l}\text { Turner et } \\
\text { al. }{ }^{29}\end{array}$ & $\begin{array}{l}{[103]} \\
28 \mathrm{NDP} \\
(\mathrm{N} / \mathrm{C}) \\
25 \mathrm{NDU} \\
(\mathrm{N} / \mathrm{C}) \\
25 \mathrm{D} \\
(\mathrm{N} / \mathrm{C}) \\
25 \mathrm{CO}\end{array}$ & $\begin{array}{l}\text { Incluídos em NDP } \\
\text { e NDU: VPT, falta } \\
\text { de sensibilidade } \\
\text { ao monofilamento } \\
\text { de } 10 \mathrm{~g} \text { (5.07) e } \\
\text { deformidades nos } \\
\text { pés. Excluídos: } \\
\text { doença musculoes- } \\
\text { quelética articular, } \\
\text { ou cirurgia no pé, } \\
\text { neuropatia perifé- } \\
\text { rica de outras cau- } \\
\text { sas, enfermidade } \\
\text { visual significante } \\
\text { ou doença arterial } \\
\text { periférica. }\end{array}$ & $\begin{array}{l}\text { 15/13NDP } \\
20 / 5 \mathrm{NDU} \\
14 / 11 \mathrm{D} \\
14 / 11 \mathrm{CO}\end{array}$ & $\begin{array}{l}64(56-71) \mathrm{NDP} \\
59(52-65) \\
\mathrm{NDU} \\
61(50-68) \mathrm{D} \\
59(52-64) \mathrm{CO}\end{array}$ & $\begin{array}{l}27,0(25,2- \\
29,1) \mathrm{NDP} \\
29,7(25,9- \\
32,4) \mathrm{NDU} \\
26,5(24,3-30,0) \\
\text { D 25,0 }(23,8- \\
30,6) \mathrm{CO}\end{array}$ & $\begin{array}{l}\text { ADM de pés } \\
\text { e índices de } \\
\text { pressão plantar } \\
\text { com palmilha } \\
\text { transdutora. }\end{array}$ & $\begin{array}{l}\text { A dorsiflexão li- } \\
\text { mitada da } 1^{a} \text { MTF } \\
\text { indica que esta é } \\
\text { mais informativa } \\
\text { para identificar } \\
\text { indivíduos com } \\
\text { aumento de } \\
\text { pressão plantar e } \\
\text { risco de úlceras, } \\
\text { quando compa- } \\
\text { rada à inversão/ } \\
\text { eversão. }\end{array}$ \\
\hline Yavuz et al. ${ }^{30}$ & $\begin{array}{l}{[35]} \\
15 \mathrm{D} \\
(10 / 5) \\
20 \mathrm{CO}\end{array}$ & $\begin{array}{l}\text { Incluídos: déficit } \\
\text { de sensibilidade } \\
\text { ao monofilamento } \\
\text { 5.07. Excluídos: } \\
\text { úlceras, grandes } \\
\text { deformidades, } \\
\text { cirurgias e dores } \\
\text { nos pés. }\end{array}$ & $\begin{array}{l}12 / 3 \mathrm{D} \\
12 / 8 \mathrm{CO}\end{array}$ & $\begin{array}{l}60,5(10,1) \mathrm{D} \\
45,8(19,8) \mathrm{CO}\end{array}$ & $\begin{array}{l}29,2(8,0) \mathrm{D} \\
24,9(3,4) \mathrm{CO}\end{array}$ & $\begin{array}{l}\text { Índices de } \\
\text { pressão plantar } \\
\text { com plataforma } \\
\text { de força. }\end{array}$ & $\begin{array}{l}\text { Indivíduos D têm } \\
\text { maior força de } \\
\text { cisalhamento na } \\
\text { planta dos pés } \\
\text { em deslocamento } \\
\text { AP e ML que os } \\
\text { CO. }\end{array}$ \\
\hline $\begin{array}{l}\text { Yavuzer et } \\
\text { al. }{ }^{31}\end{array}$ & $\begin{array}{l}{[66]} \\
20 N D P \\
(\mathrm{~N} / \mathrm{C}) \\
20 \mathrm{D} \\
(\mathrm{N} / \mathrm{C}) \\
20 \mathrm{CO}\end{array}$ & $\begin{array}{l}\text { Excluídos: úlceras, } \\
\text { pés Charcot, enfer- } \\
\text { midades neurológi- } \\
\text { cas, reumáticas, ou } \\
\text { musculares. }\end{array}$ & $\begin{array}{l}\text { 12/8NDP } \\
12 / 14 \mathrm{D} \\
10 / 10 \mathrm{CO}\end{array}$ & $\begin{array}{l}61,7(8,5) \mathrm{NDP} \\
58,2(9,5) \mathrm{D} \\
60,9(5,9) \mathrm{CO}\end{array}$ & $\begin{array}{l}29,5(4,5) \mathrm{NDP} \\
30,3(5,9) \mathrm{D} \\
28,9(5,1) \mathrm{CO}\end{array}$ & $\begin{array}{l}\text { Análise quali- } \\
\text { tativa de mar- } \\
\text { cha por meio } \\
\text { de fotogrametria } \\
\text { e software } \\
\text { específico. } \\
\text { Velocidade } \\
\text { e cadência } \\
\text { da marcha e } \\
\text { amplitude do } \\
\text { passo. }\end{array}$ & $\begin{array}{l}\text { Neuropatia } \\
\text { compromete } \\
\text { a marcha de } \\
\text { diabéticos, mas } \\
\text { características ós- } \\
\text { seas, musculares } \\
\text { e teciduais tam- } \\
\text { bém parecem } \\
\text { estar envolvidas } \\
\text { neste processo. }\end{array}$ \\
\hline
\end{tabular}

nados, respectivamente, proporção entre indivíduos do gênero masculino e feminino, idade média ou máxima e mínima e IMC distribuídos para cada grupo.

Na coluna "Parâmetros avaliados", estão descritos os métodos de avaliação para cada ensaio clínico. Estes parâmetros foram divididos em dois grandes grupos:

Parâmetros cinemáticos quantitativos (Tabela 1):
- Mensuração da velocidade, observadas em oito estudos $^{19,22-27,31}$;

- Mensuração da cadência da marcha, observada em três estudos ${ }^{19,23,31}$.

- Mensuração da amplitude do passo, observada em quatro estudos ${ }^{23,26,27,31}$;

- Variabilidade do tempo do passo, observada em três estudos $23,26,27$. 
Tabela 1. Média, Desvio-padrão e Amplitude de alguns parâmetros cinemáticos quantitativos encontrados.

\begin{tabular}{lcccccc}
\hline Variáveis & \multicolumn{2}{c}{ Média } & \multicolumn{2}{c}{ Desvio padrão } & Amplitude \\
\hline & DM & CO & DM & CO & DM & $0,90-1,45$ \\
Velocidade (m/s) & 0,87 & 1,16 & 0,17 & 0,21 & $0,70-1,25$ & $1,12-1,66$ \\
Cadência (passos/s) & 1,47 & 1,52 & 0,30 & 0,44 & $01-1,78$ \\
Amplitude do passo (m) & 0,52 & 0,59 & 0,04 & 0,08 & $0,48-0,59$ & $0,52-0,68$ \\
Variabilidade do tempo do passo (s) & 0,05 & 0,03 & 0,01 & 0,004 & $0,044-0,073$ & $0,031-0,038$ \\
Relação temporal apoio/balanço & 1,55 & 1,42 & 0,04 & 0,005 & $1,51-1,58$ & $1,42-1,43$ \\
\hline
\end{tabular}

DM: Portadores de Diabetes Mellius; CO: Controle.

- Relação temporal entre fase de apoio e fase de balanço, observada em três estudos ${ }^{22,24,25}$.

Além das variáveis dispostas na tabela 1 , foram observados, ainda, os seguintes parâmetros cinemáticos quantitativos:

- Acelerometria: Três estudos utilizaram o acelerômetro como material de coleta de dados ${ }^{23-25}$. Um estudo ${ }^{23}$ considerou a aceleração na pelve e na cabeça, além da aceleração vertical e concluiu que diabéticos, no geral, possuem uma média de aceleração 13,13\% menor nestes parâmetros quando comparados aos controles. Um estudo ${ }^{24}$ verificou a acelerometria na articulação do joelho e outro estudo ${ }^{25}$ dispôs acelerômetros nos ombros; quadris; joelhos e tornozelos, constatando, também, que a aceleração, em todas as articulações de diabéticos, encontra-se diminuída em relação aos controles.

- Tempo de ativação muscular: Quatro estu$\operatorname{dos}^{17,22,24,28}$ utilizaram o tempo de ativação muscular mensurado pela eletromiografia. Dois deles $^{22,24}$ verificaram os resultados da ativação muscular durante as fases do ciclo da marcha e verificaram atrasos de ativação em relação aos controles. $\mathrm{O}$ primeiro estudo ${ }^{17}$, com o uso de plataforma de força, verificou o tempo de ativação dos músculos vasto lateral, gastrocnêmino lateral e tibial anterior na fase de apoio, constatando atraso na ativação muscular dos dois primeiros e o segundo estudo ${ }^{28}$ verificou a ativação do vasto lateral e tibial anterior, na marcha em esteira ergométrica, e constatou atraso de ativação do dorsiflexor.

- Amplitude de movimento das articulações: Três estudos ${ }^{22,29,31}$ realizaram a medida da ADM de articulações dos membros inferiores. Dois deles $^{29,31}$ avaliaram, durante a realização da marcha: um estudo utilizou sistema de sensores, enquanto que o outro utilizou sistema de vídeo e software. Um estudo ${ }^{22}$ verificou a ADM durante o repouso, através de goniômetro. Em todas as pesquisas foi constatado que neuropatas diabéticos apresentam diminuição da ADM nos tornozelos e articulações dos pés.

\section{Parâmetros cinéticos.}

- Força de reação ao solo: Dois estudos17,18 pesquisaram a força de reação ao solo por meio de plataforma de força. $O$ primeiro estudo17 buscou relacionar as forças de reação ao solo (GRF) com a atividade muscular de determinados músculos e foi constatado que quanto maior o comprometimento sensitivo dos pés, mais comprometida se torna a fase de contato inicial e de pré-balanço devido à fraqueza da musculatura envolvida. Já o segundo estudo18 verificou as GRF, durante as fases do ciclo da marcha, e constatou que a NDP leva a um aumento da GRF relacionado com a fase de apoio, principalmente, no período da propulsão ou pré-balanço.

- Pressão plantar: Quatro estudos20,21,29,30 verificaram a distribuição de pressão nas plantas dos pés. Um deles ${ }^{30}$ utilizou plataforma de força, comparou as resultantes da força de cisalhamento em algumas regiões plantares e concluiu que diabéticos estão mais sujeitos às consequências dessa força que os controles. $O$ três demais ${ }^{20,21,29}$ utilizaram palmilha transdutora de índices de pressão. Dois deles ${ }^{20,21}$ tiveram enfoque em dispositivos para melhorar a distribuição da pressão, constatando melhora na distribuição de pressão plantar, por meio de palmilha de posicionamento ${ }^{20}$ ou de adaptação adequada no calçado $^{21}$. Já no terceiro ${ }^{29}$, que buscou correlacionar sítios de hiperpressão plantar com ADM de algumas articulações dos pés, foi observada associação significante dessas condições apenas na primeira articulação metatarsofalângica.

É sabido que diabéticos têm menor velocidade da marcha e amplitude do passo quando lhes é solicitado a deambular em uma superfície plana, em linha reta. No entanto, poucos são os estudos que 
tentam simular um padrão de marcha que se aproxime melhor daquele realizado no cotidiano. Merecem destaque os estudos ${ }^{23-27}$ que utilizam como método a locomoção em superfícies irregulares e com períodos de parada e retorno à caminhada. Esta forma de avaliação deve ser mais utilizada pela maior proximidade com a atividade real, uma vez que, em tais estudos, ficou claro que essa é a grande dificuldade na deambulação de indivíduos com NDP.

Estudos que visaram correlacionar alguns parâmetros da marcha com riscos de queda também merecem ser salientados. As alterações nervosas intrínsecas causadas pela NDP, que acabam culminando no aumento da propensão às quedas, ocorrem porque a neuropatia compromete a velocidade de condução nervosa às fibras musculares rápidas, tornando ineficaz o torque na região do tornozelo e, por conseguinte, a estabilidade médio-lateral na fase de apoio simples ${ }^{23}$.

Um dos estudos ${ }^{19}$ utilizou o expoente $\alpha$ para tentar predizer quedas pela teoria fractal (que busca uma relação entre dois processos de maneira nãolinear). No entanto, a associação entre a presença NDP com episódios de quedas e a dinâmica fractal não demonstrou significância. Dois estudos ${ }^{23,27}$ concordam que na realização da marcha sob superfície plana e boa luminosidade, os neuropatas sem restrição visual não apresentam perda de equilíbrio. Nessas condições apenas adotam um padrão mais conservador da marcha, com amplitude do passo, velocidade e cadência da marcha diminuídos. Todavia, se os locais se tornam inadequados, com superfícies irregulares e pouca luminosidade, os indivíduos passam a ter maiores desequilíbrios e dificuldade de deambulação.

Um estudo ${ }^{26}$ realizou intervenção com diferentes tipos de auxílio, tais como bengala, apoio em uma superfície vertical (parede) e órteses, durante a execução da marcha de neuropatas. Este estudo concluiu que os auxílios utilizados melhoram a regularidade da marcha sob condições irregulares e baixa luz. Esta intervenção pode permitir aos pacientes manter a otimização da marcha e diminuir a propensão às quedas perante circunstâncias difíceis.

Em relação à atividade muscular alguns estu$\operatorname{dos}^{17,18,22,28}$ concordam com a disfunção na ativação do tríceps sural na fase de apoio terminal e prébalanço. Segundo esses autores, essa alteração faz com que, no final da fase de apoio, momento em que o tríceps age de maneira concêntrica para impulsionar o pé contra o solo, não haja a flexão plantar esperada. Essa situação encurta a fase de balanço, pois o membro não impulsionado adequadamente, tocará prematuramente o solo no próximo apoio, diminuindo a absorção de impactos, gerando défi- cits no equilíbrio e progressão da marcha.

Dois estudos ${ }^{20,21}$ verificaram a eficácia da utilização de palmilhas e calçado específico para diminuição da hiperpressão plantar de diabéticos com NDP. $\mathrm{O}$ primeiro ${ }^{20} \mathrm{fez}$ uso de uma palmilha com aumento do arco plantar e o segundo ${ }^{21}$ utilizou um calçado com mecanismo "rocker-sole" (elevação de determinadas regiões da sola do sapato, visando aliviar as áreas de hiperpressão plantar). Ambos concluíram que a adoção destes utensílios levou à diminuição da pressão das regiões plantares envolvidas.

\section{CONCLUSÃO}

Foi possível notar que, para a elaboração do perfil da amostra e distribuição dos indivíduos nos grupos, são muitas as ferramentas utilizadas para a confirmação diagnóstica da NDP desde questionários validados, exames clínicos simples até os mais sofisticados. Em geral, todas essas ferramentas foram eficazes para discriminar o(s) grupo(s) neuropata(s) dos não-neuropata(s), devido às diferenças significantes observadas nas variáveis coletadas. Contudo, dentro desse parâmetro, nota-se uma falta de registro do nível de atividade física dos participantes, o que pode influenciar nos resultados obtidos. Além disso, não é comum encontrar a descrição do grau de comprometimento pela NDP entre os indivíduos, fato que também pode se tornar um parâmetro de confusão na interpretação dos resultados. Alguns poucos estudos relatam apenas o tempo de diagnóstico de DM, porém este pode ser um dado um tanto vago, pois se o enfermo está em tratamento, a progressão da NDP pode ser retardada.

Em síntese, parece que a NDP leva a déficits na amplitude do passo, velocidade e cadência da marcha, em condições normais (terreno plano, linha reta e velocidade constante), além de déficits de equilíbrio e coordenação, em condições especiais (aclives, declives e terrenos irregulares). Além disso, os neuropatas apresentam dificuldade na fase de apoio terminal e pré-balanço devido à alteração de ativação do tríceps sural e contato inicial adequado com o antepé, sem absorção de choques. Pode ser observado, ainda, aumento nos índices de pressão plantar devido à própria patogenia da NDP associada aos mecanismos intrínsecos do descontrole glicêmico, como comprometimento ósseo e tecidual.

Indagações sobre exato mecanismo de quedas e como contribuir para preveni-las, ainda deverão ser alvo de futuras pesquisas. Deve-se buscar, nas próximas pesquisas, correlacionar os fatores relacionados aos riscos de quedas - velocidade e cadência da marcha, 
variabilidade e amplitude do passo, relação temporal entre fase de apoio e balanço e acelerometria - pesquisados por grande parte dos autores, com a atividade muscular, bem como, com os picos de pressão plantar nas diversas fases da marcha em condições normais e em condições especiais. Atividades que incluam a prática do cotidiano ou da vida real devem ser inseridas, tanto em simulações para realização de pesquisas, quanto para se traçar estratégias de tratamento, uma vez que se sabe que o padrão de deambulação dos portadores de NDP é dificultado por essas condições.

\section{REFERÊNCIAS BIBLIOGRÁFICAS}

1. Wild S, Roglic G, Green A, Sicree R, King H. Global prevalence of diabetes: estimates for the year 2000 and projections for 2030. Diabetes Care 2004;27(5):1047-1053.

2. Gregg EW, Paulose-Ram R, Gu Q, Eberhardt MS, Wolz $M$, Burt V, et al. Prevalence of Lower-Extremity Disease in the U.S. adult population $>40$ years of age with and without diabetes. Diabetes Care 2004;27(7):1591-1597.

3. Vinik AI, Park TS, Stansberry KB, Pittenger GL. Diabetic neuropathies. Diabetologia 2000;43(8):957-973.

4. Boulton AJM, Malik RA, Arezzo JC, Sosenko JM. Diabetic Somatic Neuropathies. Diabetes Care 2004:27(6):458-486.

5. Andersen H, Gadeberg PC, Brock B, Jakobsen J. Muscular atrophy in diabetic neuropathy: a stereological magnetic resonance imaging study. Diabetologia 1997;40(9):1062-1069.

6. Porciúncula MVP, Rolim LCP, Garofolo L, Ferreira SRG. Análise de fatores associados à ulceração de extremidades em indivíduos diabéticos com neuropatia periférica. Arq Bras Endocrinol Metabol 2007;51(7):1134-1142.

7. Giacomozzi C, Caselli A, Macellari V, Giurato L, Lardieri L, Uccioli L. Walking Strategy in Diabetic Patients With Peripheral Neuropathy. Diabetes Care 2002;25(8):1451-1457

8. Sacco ICN, Amadio AC. A study of biomechanical parameters in gait analysis and sensitive cronaxie of diabetic neuropathic patients. Clin Biomech 2000;15(3):196-200.

9. Witzket KA, Vinik AI. Diabetic Neuropathy in Older Adults. Rev Endocr Metab Disord 2005;6(2):117-127.

10. Freiberger E, Menz HB. Characteristics of falls in physically active community-dwelling older people. Z Gerontol Geriat 2006;39(4):261-267.

11. Hollman JH, Kovash FM, Kubik JJ, Linbo RA. Agerelated differences in spatiotemporal markers of gait stability during dual task walking. Gait Posture 2007;26(1):113-119.

12. Alfieri FM. Distribuição da pressão plantar em idosos após intervenção proprioceptiva. Rev Bras Cineantropom Desempenho Hum 2008;10(2):137-142.

13. Kanade RV, VanDeursen RWM, Harding K, Price P. Walking performance in people with diabetic neuropathy: benefits and threats. Diabetologia 2006;49(8):1747-1754.

14. Gutierrez EM, Helber MD, Dealva D, Ashton-Miller JA, Richardson JK. Mild diabetic neuropathy affects ankle motor function. Clin Biomech 2001;16(6):522-828.

15. Wallace C, Reiber GE, LeMaster J, Smith DG, Sullivan K, Hayes S, Vath C. Incidence of falls, risk factors for falls, and fall-related fractures in individuals with diabetes and a prior foot ulcer. Diabetes Care 2002;25(11):1983-1986.
16. Maurer MS, Burcham J, Cheng H. Diabetes mellitus is associated with an increased risk of falls in elderly residents of a long-term care facility. J Gerontol 2005;60(9):1157-1162.

17. Akashi PMH, Sacco ICN, Watari R, Hennig E. The effect of diabetic neuropathy and previous foot ulceration in EMG and ground reaction forces during gait. Clin Biomech 2008;23(5):584-592.

18. D’Ambrogi E, Giacomozzi C, Macellari V, Uccioli L. Abnormal foot function in diabetic patients: the altered onset of Windlass mechanism. Diabet Med 2005;22(12):1713-1719.

19. Gates DH, Dingwell JB. Peripheral neuropathy does not alter the fractal dynamics of stride intervals of gait. J Appl Physiol 2007;102(3):965-971.

20. Guldemond NA, Leffers P, Schaper NC, Sanders AP, Nieman F, Willems P, Walenkamp GHIM. The effects of insole configurations on forefoot plantar pressure and walking convenience in diabetic patients with neuropathic feet. Clin Biomech 2007;22(1):81-87.

21. Hsi WL, Chai HM, Lai JS. Evaluation of rocker sole by pressure-time curves in insensate forefoot during gait. Am J Phys Med Rehabil 2004;83(7):500-5-6.

22. Kwon OY, Minor SD, Maluf KS, Mueller MJ. Comparsion of muscle activity during walking in subjects with and without diabetic neuropathy. Gait Posture 2003;18(1):105-113.

23. Menz HB, Lord SR, St George R, Fitzpatrick RC. Walking stability and sensorimotor function older people with diabetic peripheral neuropathy. Arch Phys Med Rehabil 2004;85(2):245-252.

24. Petrofsky JS, Lee S, Cuneo ML. Gait characteristics in patients with type 2 diabetes; improvement after administration of rosiglitazone. Med Sci Monit 2005;11(6):PI43-P1451.

25. Petrofsky JS, Lee S, Bweir S. Gait characteristics in people with type 2 diabetes mellitus. Eur J Appl Physiol 2005;93(5-6):640-647.

26. Richardson JK, Thies SB, DeMott TK, Ashton-Miller JA. Interventions improve gait regularity in patients with peripheral neuropathy while walking on an irregular surface under low light. A Am Geriatr Soc 2004;52(4):510-515.

27. Richardson JK, Thies SB, DeMott TK, Ashton-Miller JA. Gait analysis in a challenging environmental differentiates between fallers and nonfallers among older patients with peripheral neuropathy. Arch Phys Med Rehabil 2005;86(8):1539-1544.

28. Sacco ICN, Amadio AC. Influence of the diabetic neuropathy on the behavior of electromyographic and sensorial responses in treadmill gait. Clin Biomech 2003;18(5):426-434.

29. Turner DE, Helliwell PS, Burton AK, Woodburn J. The relationship between passive range of motion and range of motion during gait and plantar pressure measurements. Diabet Med 2007;24(11):1240-1246.

30. Yavuz M, Tajaddini A, Botek G, Davis BL. Temporal characteristics of plantar shear distribuition: Relevance to diabetic patients. J Biomech 2008;41(3):556-559.

31. Yavuzer G, Yetkin I, Toruner FB, Koca N, Bolukbas $\mathrm{N}$. Gait deviations of patients with diabetes mellitus: looking beyond peripheral neuropathy. Eura Medicophys 2006; 42(2):127-133.

\section{Endereço para correspondência}

Marcela Regina de Camargo

Rua José Manoel Fonseca, 97, Vila Euclides, CEP 19014-050 - Presidente Prudente SP, Brasil E-mail: amy.marcela@gmail.com 